\title{
Formas de afrontamiento y afectividad en personas con VIH/SIDA. Diferencias entre toxicómanos y no toxicómanos
}

\author{
Pernas, A. ${ }^{1+}$; Iraurgi, l. ${ }^{2}$; Bermejo, P. $^{1}$; Basabe, N. ${ }^{3}$; Carou, M. ${ }^{1}$; Paez, D. ${ }^{3}$; Cabarcos, A $^{4}$. \\ (1) Unidade Municipal de Atencion a Drogodependentes. Santiago de Compostela. \\ (2) Módulo de Asistencia Psicosocial de Rekalde. \\ (3) Dpto. Psicología Social - Universidad del País Vasco. \\ (4) Servicio de Medicina Interna, Hospital General de Galicia. \\ t.- In Memorian \\ Enviar correspondencia a: \\ loseba Iraurgi Castillo. Módulo de Asistencia Psicosocial de Rekalde. C/ Camilo Villabaso 24 lonja. 48002 Bilbao. E-mail: iraurgi@euskalnet.net.
}

\section{RESUMEN}

OBJETIVO.- Examinar las diferencias en las formas de afrontamiento de la seropositividad a VIH entre personas toxicómanas y no toxicómanas afectadas, y explorar la relación entre las formas de afrontamiento de la seropositividad y la afectividad. DISEÑO.- Transversal, comparación de dos grupos. MÉTODO.- 105 sujetos seropositivos a VIH (50 toxicómanos y 55 no-toxicómanos) son evaluados a través de un cuestionario que agrupa escalas de afrontamiento de la seropositividad, variables del estado de ánimo (depresión, ansiedad y afectividad) y el apoyo social objetivo y subjetivo. RESULTADOS.- No se encuentran diferencias en la forma de afrontar la seropositividad entre los grupos, ni tampoco respecto a la afectividad. Un análisis correlacional muestra que las formas de afrontamiento activas, tanto cognitivas como conductuales, están asociadas $(p<0,05)$

una mayor afectividad positiva $(r=0,45)$ y a una menor ansiedad $(r=-0,24)$, mientras las formas evitantes están asociadas a una mayor depresión ( $r=0,31)$, afectividad negativa $(r=0,31)$, ansiedad rasgo $(r=0,33)$, y ansiedad estado $(r=0,21)$. CONCLUSIÓN.Los resultados del estudio guardan concordancia con los encontrados en la literatura, que muestran una relación positiva entre las formas activas y la afectividad, así como una asociación negativa de ésta con las formas evitantes.

Palabras claves: VIH, Afectividad, Afrontamiento, Adicción a drogas.

\section{SUMMARY}

OBJECTIVES.- Differences in coping with HIV-infection between drug users and no-drug users were examined, and the relationship between coping and affectivity were explored. METHOD.- 105 HIV-infected subjects (50 drug users and 55 no-drug users) were evaluated in a cross-over study. Participants completed self-report measures including coping strategies with HIV-infection, mood state / affectivity variables (depression, anxiety and positive / negative affect) and the objective and subjective social support. RESULTS.- No differences were found in the coping forms between groups, nor regarding the affectivity. A correlational analysis showed that active coping, behavioural and cognitive, were associated $(p<0,05)$ with higher positive affect $(r=0,45)$ and lower anxiety $(r=-0,24)$, while avoidance coping were associated with higher levels of depression $(r=0,31)$, negative affect $(r=0,31)$, trait anxiety $(r=$ $0,33)$, and state anxiety $(r=0,21)$. CONCLUSION.-The results of the study are concordant with found in the literature, that show a positive relationship between active coping forms and affectivity, as well as a negative association of this with avoidance coping.

Key words: HIV, Affectivity, Coping, Drug Addiction.

\section{INTRODUCCIÓN}

er portador del VIH o enfermo de SIDA son situaciones que generan un conjunto multifacético de estresores que frecuentemente exceden las capacidades de afrontamiento que los afectados poseían antes de que apareciese la enfer- medad. Asimismo, la vía de infección también podría concurrir con estresores y consecuencias diferenciales. Por ejemplo, entre los toxicómanos, además de la amenaza del VIH/SIDA, se añaden otras posibles infecciones y una situación de mayor deterioro físico y social. Entre los homosexuales, además del estigma que ya existe en la sociedad sobre este colectivo, la 
infección por VIH/SIDA puede llevar a estas personas a ser también estigmatizadas desde dentro de su propio grupo. Estas situaciones frecuentemente tienen un impacto adverso sobre el funcionamiento personal y social, el autoconcepto y estado emocional, la capacidad física, las relaciones familiares e interpersonales y la actividad sexual (Kalichman y Sikkema, 1994).

Un afrontamiento exitoso de la situación de seropositividad y/o enfermedad puede tener un impacto sobre el estado físico, inmunológico y afectivo del sujeto, sobre la longitud y curso de su supervivencia, así como en el área social y de manejo de roles (Basabe, Iraurgi, Paez e Insua, 1996). La ya clásica definición de Lazarus y Folkman del afrontamiento nos situaría ante "aquellos esfuerzos cognoscitivos y/o conductuales constantemente cambiantes que se desarrollan para manejar las demandas específicas externas y/o internas que son evaluadas como excedentes o desbordantes de los recursos del individuo" (Lazarus y Folkman, 1986, pg. 164). En el caso de la enfermedad, las formas de afrontamiento más adaptativas ya han sido descritas en la literatura (Taylor, 1983; Basabe, Valdoseda y Paez, 1993) y son: buscar el significado del evento, intentar dominar la situación, y los esfuerzos para mejorar la autoestima. Entre las formas de afrontamiento perjudiciales para la salud, se han descrito los estilos represivo y de inhibición (Paez, 1993). El estilo represivo de afrontamiento se caracterizaría por la evitación y la no confrontación de los hechos estresantes a la vez que se inhibe la reacción emocional concomitante.

Nuestro objetivo en este trabajo es doble. Por una parte, examinar las diferencias en las formas de afrontamiento de la seropositividad a VIH entre personas toxicómanas y no toxicómanas afectadas y, en segundo lugar, explorar la relación entre las formas de afrontamiento de la seropositividad y la afectividad.

\section{MÉTODO}

Diseño y Muestra.- Estudio transversal sobre 105 sujetos: 50 toxicómanos en tratamiento en un Centro de atención a las drogodependencias; y 55 sujetos no toxicómanos usuarios de un servicio de asistencia a personas afectadas por VIH. La selección de los sujetos fue incidental. Al grupo de toxicómanos se les pidió colaboración en el momento que acudían a revisión al Servicio de Medicina Interna del Hospital General de Galicia completando un cuestionario de autoinforme. El grupo de no toxicómanos eran voluntarios que iniciaban un programa de intervención psicosocial y manejo de estrés en un Centro de Asistencia a personas Infectadas por VIH, contestando a un cuestionario equivalente al ofrecido a la otra muestra, y constituyendo la línea base de la evalua- ción del programa que iniciaban (Iraurgi, Basabe, Sastre et al, 1996).

Todos los sujetos recibían asistencia sanitaria por sus problemas médicos. El grupo de toxicómanos, con diagnóstico de adicción a opiáceos, además del seguimiento por su infección a VIH, estaba incluido en un programa de deshabituación a drogas.

Instrumentos.- Todos los sujetos fueron evaluados con un cuestionario que recogía información sobre:

1. Medida de afrontamiento. Se ha utilizado la escala de 47 ítems propuesta por Namir y colaboradores (1987). La escala evalúa respuestas cognitivas y conductuales como formas de afrontar la seropositividad. Siguiendo las indicaciones de las autoras, se obtuvieron tres métodos o dimensiones de afrontamiento: 1) activo-cognitivo, que recoge la evaluación de la fuente de estrés (la enfermedad) e incluye aspectos sobre las creencias, actitudes y pensamientos sobre la forma de conducirse con la seropositividad [fiabilidad mediante el alpha de Cronbach $(\alpha)$ de 0,70$], 2)$ afrontamiento activoconductual $[\alpha=0,83]$, el cual implica un comportamiento directo sobre el problema y la búsqueda de apoyo emocional, informativo e instrumental; y 3) el método de evitación [ $\alpha=$ $0,76]$, que haría alusión a la no confrontación del problema a través de estrategias de negación o evitar pensar sobre ello, o a través de comportamiento de anestesia cognitiva mediante el uso de alcohol y drogas. Por otra parte, estos tres métodos se analizan de forma más específica a través de ocho estrategias: a) participación activa-positiva $[\alpha=0,81]$, b) búsqueda de información y expresión activa $[\alpha=0,68]$, c) relación activa con otros $[\alpha=0,67], d)$ crecimiento personal y comprensión positiva $[\alpha=0,61]$, e) rumiación y/o cognición pasiva $[\alpha=0,56]$, f) distracción $[\alpha=0,18], g)$ resignación pasiva $[\alpha=$ $0,56]$, y h) evitación o conductas pasivas $[\alpha=$ $0,67]$. Tanto las dimensiones de afrontamiento como las estrategias han sido calculadas mediante un algoritmo que permite la homogeneidad y comparación entre las mismas, oscilando la escala de medida entre cero y cuatro.

2. Valoración del estado afectivo. Para su estimación se utilizó una batería de escalas que comprenden: 1) el STAI - Cuestionario de Ansiedad Estado-Rasgo (Spielberger, Gorsuch y Lushene, 1970), con una fiabilidad de 0,92 y 0,86 para las dimensiones correspondientes, 2) la Escala de Depresión de Hamilton (en Conde y Franch, 1984), con un alpha de 0,91, y 3) el PANAS Escala de Afectividad Positiva y Negativa de Watson, Clark y Tellegen (1988). Mediante esta última escala se busca medir el grado de malestar/bienestar subjetivo (afectividad negati- 
va y positiva). Asimismo, se ha obtenido la variable "balanza de afectos" como resultado de la diferencia entre el afecto positivo y el negativo. Para la escala total se ha obtenido una fiabilidad de 0,79; para la dimensión de afectividad positiva un $\alpha=0,78$; y para el afecto negativo un $\alpha=0,82$.

3. Escala de apoyo social subjetivo de Vaux y colaboradores (1986) y de apoyo objetivo del Dpto. de Salud Mental de California (Paez, 1986). La fiabilidad alcanzada por ambas escalas fue de 0,82 y 0,70 , respectivamente.

Análisis estadísticos.- Se llevó a cabo un primer análisis descriptivo y del comportamiento de las variables calculándose los estadísticos de tendencia central (Media -M- y Desviación Estándar -DE-) en el caso de variables continuas, y de proporciones para las variables discretas. Asimismo, se estimó la fiabilidad en la muestra de las escalas utilizadas mediante el coeficiente alpha de Cronbach.
Para contrastar el primer objetivo, se examinaron las diferencias entre los grupos respecto a las variables de afectividad y formas de afrontamiento a través de análisis de varianza. La relación entre estos dos conjuntos de variables, afectividad y afrontamiento, se estimó a través del coeficiente de correlación de Pearson (r). Los análisis fueron realizados con el programa SPSS en su versión 6.1 (Norusis, 1994)

\section{RESULTADOS}

Los sujetos eran prioritariamente hombres (80\%) con una edad media de 29,9 años (DE: 8,8). El tiempo medio de conocimiento de la seropositividad era de 3,2 años (rango: 10-0 años); todos ellos se mostraban asintomáticos si bien, en el momento del estudio, recibían tratamiento antirretroviral un $24 \%$ del grupo de toxicómanos y un $25,4 \%$ de los no toxicómanos. Ambos grupos eran homogéneos respecto a las variables sociodemográficas y de curso de la infección.

Tabla 1. Formas de afrontamiento ante la seropositividad. Diferencias por grupos

\begin{tabular}{|c|c|c|c|c|c|c|c|}
\hline & & $\mathbf{M}_{\text {Tot }}$ & $\mathbf{M}_{\text {Tox }}$ & $\mathbf{M}_{\text {Ntox }}$ & $\mathbf{F}$ & g.l. & $\mathbf{p}$ \\
\hline \multicolumn{8}{|l|}{ Dimensiones } \\
\hline Activo cognitivo & & 2,67 & 2,68 & 2,67 & 0,04 & 1,103 & 0,89 \\
\hline Activo conductual & & 2,54 & 2,47 & 2,60 & 1,76 & 1,103 & 0,19 \\
\hline Evitación & & 2,40 & 2,45 & 2,35 & 0,89 & 1,103 & 0,35 \\
\hline \multicolumn{8}{|l|}{ Estrategias } \\
\hline Crecimiento personal & & 2,69 & 2,68 & 2,70 & 0,03 & 1,103 & 0,87 \\
\hline Implicación activa & & 2,50 & 2,44 & 2,56 & 1,22 & 1,103 & 0,27 \\
\hline Expresión activa & & 2,51 & 2,43 & 2,58 & 1,31 & 1,103 & 0,25 \\
\hline Relación con otros & & 2,56 & 2,43 & 2,68 & 2,75 & 1,103 & 0,10 \\
\hline Distracción & & 2,30 & 2,25 & 2,34 & 0,60 & 1,103 & 0,44 \\
\hline Resignación pasiva & & 2,69 & 2,78 & 2,62 & 2,63 & 1,103 & 0,11 \\
\hline Rumiación & & 2,67 & 2,83 & 2,53 & 3,46 & 1,103 & 0,06 \\
\hline Evitación & & 2,26 & 2,33 & 2,19 & 0,52 & 1,103 & 0,47 \\
\hline $\begin{array}{ll}M_{\text {Tot }} & \text { Media Total } \\
M_{\text {Tox }} & \text { Media Toxicómanos } \\
M_{\text {Ntox }} & \text { Media No Toxicómanos }\end{array}$ & $\begin{array}{l}\text { F - Prueba de Snedecor } \\
\text { gl - Grados de Libertad } \\
\text { p - Significación }\end{array}$ & & & & & & \\
\hline
\end{tabular}

El análisis de la utilización de las formas de afrontamiento en función del uso o no de drogas (Tabla 1) no muestra, para el conjunto de dimensiones y estrategias, diferencias estadísticamente significativas $(p<0,05)$, indicando una homogeneidad en la forma de afrontar la seropositividad en toxicómanos o no-toxicómanos. Asimismo, no se hayan diferencias en las variables afectivas (Tabla 2).

Un análisis correlacional (Tabla 3) muestra que las formas de afrontamiento activas, tanto cognitivas como conductuales, están asociadas $(p<0,05)$ a una mejor afectividad (mayor afecto positivo, $r=0,45$; menor ansiedad, $r=-0,24)$, mientras las formas evitantes están asociadas a una peor afectividad (mayor depresión, $r=0,31$; mayor afectividad negativa, $r=$ 0,31 ; y mayor ansiedad rasgo, $r=0,33$ y estado, $r=$ $0,21$,$) .$

Más específicamente y revisando la relación entre las diversas estrategias de afrontamiento y el estado emocional (Tabla 3), un estado afectivo positivo se relaciona con el crecimiento personal $(r=0,45)$, la implicación afectiva $(r=0,35)$, la expresión emocional activa $(r=0,27)$, la relación con otros $(r=0,35)$, y de forma tendencial con la distracción $(r=0,18, p<0,10)$. Congruentemente con lo anterior una menor depresión está asociada a un mayor crecimiento personal $(r=-0,22)$ y a una mayor relación hacia los otros ( $r=-$ $0,22)$. Por el contrario, el estado afectivo negativo no 
Tabla 2. Variables psicosociales. Diferencias por grupos.

\begin{tabular}{|c|c|c|c|c|c|c|}
\hline & $\mathbf{M}_{\text {Tot }}$ & $\mathbf{M}_{\text {tox }}$ & $\mathbf{M}_{\text {Ntox }}$ & $\mathbf{F}$ & g.l. & $\mathbf{p}$ \\
\hline Ansiedad estado (0-60) & 26,91 & 26,51 & 27,39 & 0,23 & 1,100 & 0,63 \\
\hline Ansiedad rasgo (0-60) & 28,49 & 27,32 & 29,85 & 3,39 & 1,100 & 0,07 \\
\hline Afectividad positiva (10-50) & 31,09 & 31,67 & 30,39 & 0,82 & 1,99 & 0,36 \\
\hline Afectividad negativa (10-50) & 30,16 & 31,47 & 28,59 & 3,56 & 1,99 & 0,06 \\
\hline Balanza de afectos (-40-40) & 1,08 & 0,20 & 2,21 & 1,20 & 1,96 & 0,31 \\
\hline Depresión (11-55) & 42,47 & 40,67 & 44,73 & 2,05 & 1,98 & 0,15 \\
\hline Apoyo Social Objetivo (5-20) & 11,28 & 10,98 & 11,60 & 1,05 & 1,103 & 0,31 \\
\hline Apoyo Social Subjetivo (10-40) & 29,81 & 29,93 & 29,69 & 0,04 & 1,101 & 0,85 \\
\hline $\begin{array}{ll}M_{\text {Tot }} & \text { Media Total } \\
M_{\text {Tox }} & \text { Media Toxicómanos } \\
M_{\text {Ntox }} & \text { Media No Toxicómanos }\end{array}$ & $\begin{array}{l}\text { F - Prueba de Snedecor } \\
\text { gl - Grados de Libertad } \\
\text { p - Significación }\end{array}$ & & & & & \\
\hline
\end{tabular}

Tabla 3. Asociación entre las formas de afrontamiento y la afectividad y apoyo social ( $n=105)$.

\begin{tabular}{|c|c|c|c|c|c|c|c|c|c|}
\hline & & AR & $\mathrm{AE}$ & A+ & A- & Bal & D & ASO & ASS \\
\hline \multicolumn{10}{|c|}{ Dimensiones } \\
\hline \multicolumn{2}{|c|}{ Activo cognitivo } & -.03 & -.03 & $.31^{* *}$ & .11 & .14 & -.03 & -.01 & .11 \\
\hline \multicolumn{2}{|r|}{ Activo conductual } & -.10 & -.07 & $.41^{* * *}$ & .09 & $.23^{*}$ & -.05 & .11 & $.26^{* *}$ \\
\hline \multicolumn{2}{|r|}{ Evitación } & $.33^{* * *}$ & $.21^{*}$ & .06 & $.31^{* * *}$ & $-.22^{*}$ & $.31^{* *}$ & -.01 & -.02 \\
\hline \multicolumn{10}{|c|}{ Estrategias } \\
\hline \multirow{2}{*}{\multicolumn{2}{|c|}{$\begin{array}{l}\text { Crecimiento personal } \\
\text { Implicación activa }\end{array}$}} & $-.24^{*}$ & -.19 & $.45^{* * *}$ & -.06 & $.39^{* * *}$ & $-.22^{*}$ & .06 & $.22^{*}$ \\
\hline & & -.04 & -.06 & $.35^{* * *}$ & .08 & $.20^{*}$ & .02 & -.01 & .15 \\
\hline \multicolumn{2}{|r|}{ Expresión activa } & -.05 & .07 & $.27^{* *}$ & .10 & .11 & -.06 & .16 & $.26^{* *}$ \\
\hline \multicolumn{2}{|c|}{ Relación con otros } & -.12 & -.12 & $.35^{* * *}$ & .08 & $.19^{8}$ & $-.22^{*}$ & $.31^{* *}$ & $.26^{* *}$ \\
\hline \multicolumn{2}{|c|}{ Distracción } & .07 & .11 & $.18^{\&}$ & $.24^{*}$ & -.06 & .05 & .08 & .07 \\
\hline \multicolumn{2}{|c|}{ Resignación pasiva } & $.22^{*}$ & .10 & -.08 & $.24^{*}$ & $-.26^{* *}$ & $.29^{* *}$ & $-.20^{*}$ & -.07 \\
\hline \multicolumn{2}{|c|}{ Rumiación } & $.27^{* *}$ & .16 & .02 & $.20^{*}$ & -.13 & $.23^{*}$ & -.03 & -.11 \\
\hline \multicolumn{2}{|c|}{ Evitación } & $.38^{* * *}$ & $.31^{* * *}$ & .02 & $.28^{* *}$ & $-.22^{*}$ & $.26^{* *}$ & .16 & .06 \\
\hline \multirow{4}{*}{\multicolumn{3}{|c|}{$\begin{array}{ll}* * * & p<0,001 \\
* * & p<0,01 \\
* & p<0,05 \\
\& & p<0,10\end{array}$}} & \multicolumn{3}{|c|}{ Bal - Balanza de afectos } & & & & \\
\hline & & & $\mathrm{D}-$ & Depresión & & & & & \\
\hline & & & ASO - & Apoyo Soci & Objetivo & & & & \\
\hline & & & ASS - & Apoyo Soci & Subjetivo & & & & \\
\hline
\end{tabular}

se relaciona con las estrategias activas ni con las de aproximación a los otros sino que se relaciona con estrategias de evitación y distanciamiento, en concreto, se asocia a la evitación $(r=0,28)$, la resignación pasiva $(r=0,24)$, la rumiación $(r=0,20)$ y la distracción $(r=0,24)$. La ansiedad presenta un patrón similar al anterior relacionándose principalmente con las formas evitantes: la ansiedad rasgo se asocia a la evitación $(r=0,38)$, a la rumiación $(r=0,27)$ y a la resignación pasiva; por otro lado, también se relaciona con un menor crecimiento personal $(r=-0,24)$. La ansiedad estado sólo presenta asociación estadísticamente significativa con la evitación $(r=0,31)$.

Una mejor balanza de afectos (recordar que se trata de la diferencia entre estado afectivo positivo y el negativo) se relaciona con más afrontamiento activo (mayor crecimiento personal $r=0,39$, implicación activa $r=0,20$ y relación con otros $r=0,19)$, y menor evitación $(r=-0,22)$ y menor resignación pasiva $(r=-0,26)$.
Por último, el apoyo social subjetivo se asocia a un mayor afrontamiento activo conductual $(r=0,26)$, y en concreto a la expresión emocional $(r=0,26)$ y a la relación activa con otros $(r=0,26)$, ya un mayor crecimiento personal $(r=0,22)$. El apoyo social objetivo, las redes sociales de la persona, presenta menor número de relaciones, se asocia a una mayor búsqueda de las relaciones con los otros $(r=0,31)$ y a una menor resignación pasiva $(r=-0,20)$.

\section{DISCUSIÓN}

Un primer hallazgo encontrado en este estudio es la no diferencia en la forma de afrontar la seropositividad entre personas toxicómanas y las no-toxicómanas. Tan sólo en la estrategia de rumiación se observa una diferencia tendencial $(p=0,06)$, indicándose una 
mayor utilización por parte del grupo de toxicómanos. Lo que en el quehacer diario pudiera parecer evidente, los datos lo recusan. Así, por ejemplo, se esperaba un mayor uso de drogas por parte del grupo toxicómano (estrategia de evitación) y el resultado no confirma esta hipótesis. Existen dos posibles explicaciones para este efecto. La primera que los toxicómanos están en proceso de deshabituación y debido al tratamiento se produce una reducción del uso de tóxicos; la segunda implicaría que el grupo de no-toxicómanos fuera el que incrementa el uso de drogas (alcohol, estimulantes, etc.) equiparándose entonces al grupo de drogodependientes. Por otra parte, este resultado de no diferencias podría ser debido a la utilización de cuestionarios cerrados que en muchas ocasiones ciegan o distorsionan las realidades de los distintos grupos. Una posibilidad de solventar esta limitación podría radicar en complementar estos estudios con análisis de tipo más cualitativo.

En cualquier caso, observamos que en el conjunto de la muestra, los sujetos seropositivos utilizan en mayor medida métodos activos-cognitivos, seguido por formas activas-conductuales $y$, en menor medida, por formas evitantes. Más específicamente, entre las estrategias más utilizadas se encuentran el crecimiento personal, la resignación y la rumiación; la primera de ellas considerada como adaptativa (Paez, 1993) mientras las otras dos se han asociado a estados afectivos negativos. Por otro lado, la evitación y la distracción son las estrategias menos utilizadas, lo cual es alentador si consideramos que la no confrontación suele estar asociada a una peor adaptación y progresión de la enfermedad.

Respecto al estado emocional, tampoco se han encontrado diferencias significativas entre los grupos, si bien se ha observado, de forma tendencial, una mayor ansiedad rasgo entre los no-toxicómanos y una mayor afectividad negativa entre los toxicómanos. Esto último podría ser debido a los problemas colaterales que supone una toxicomanía y el proceso de deshabituación. En general, la ansiedad evidenciada por el conjunto de la muestra es moderada (puntuaciones de 26,91 en ansiedad estado y 28,49 en ansiedad rasgo sobre un máximo de 60), si bien se encuentra altos niveles de depresión (una puntuación de 42,47 sobre un máximo de 55).

Por último, los resultados del estudio son concordantes con los ya encontrados por otros autores (véase revisión de Basabe et al, 1996; Iraurgi y Jiménez-Lerma, 1998) que muestran una relación positiva entre las formas activas y la afectividad, así como una asociación negativa de ésta con las formas evitantes. Los métodos de afrontamiento activos, tanto el cognitivo como el conductual se asocian a una mayor afectividad positiva y/o a una mejor balanza de afecto; en cambio, el afrontamiento de evitación no sólo se relaciona con una peor afectividad, sino también con una mayor ansiedad y depresión. Más específicamente, las estrategias de resignación pasiva y evitación, y sobre todo esta última, se asocian a un peor estado anímico, mientras el crecimiento personal y la relación con otros conllevan una mayor animosidad contribuyendo, quizás, a una disminución de estados depresivos. Coherentemente con resultados anteriores de otros estudios (Echevarría y Paez, 1989), la afectividad positiva se relaciona con las formas de aproximación a los otros, con la búsqueda de apoyo social lo que facilitaría la expresión emocional, por el contrario, la afectividad negativa y la ansiedad (asociada a ella) conduce a una respuesta de evitación y distanciamiento del problema o agente estresante. Estas diferentes formas de respuesta ante el afecto positivo versus negativo pueden expresar la especificidad de respuestas y estados afectivos.

En definitiva, los datos parecen apuntar hacia los métodos activos, en general, y las estrategias de crecimiento personal, relación con otros y expresión activa como las formas más adaptativas; siendo la evitación y la resignación pasiva las más distorsionadoras.

La relación entre apoyo social y formas de afrontamiento, parecen indicar que efectivamente el apoyo social puede funcionar como una forma de asistencia al afrontamiento del problema, o que el apoyo social podría actuar como paraguas protector para afrontar el problema. Así, la relación entre apoyo social subjetivo (recuérdese que es éste tipo de apoyo el que tiene un mayor impacto en el estado emocional) y afrontamiento indica que las personas que buscan la relación con otros para entender el problema, para expresar sus emociones ante el mismo y que afrontan dicha situación como una forma de crecimiento personal (de forma activa y positiva) siente asimismo que poseen una red social de amigos y familiares que les apoyan y con la que se sienten vinculados.

Enfrentarse a una enfermedad de amenaza vital, como es el SIDA, requiere habilidades de afrontamiento que puedan involucrar una reevaluación de la situación en términos más positivos, o la implicación activa para cometer las realidades de la situación tomando acciones apropiadas.

\section{AGRADECIMIENTOS}

Este estudio ha sido posible gracias a las Becas UPV-109.231-EC196/95 y GV-109.231-0007/94 (PI94/ 07) del Gobierno Vasco y a una Beca de la Conselleria de Sanidade e Servicios Sociais de la Xunta de Galicia.

\section{IN MEMORIAN}

A la memoria de Ana Pernas; nuestra amiga, nuestra compañera de trabajo. Su recuerdo, a veces, llena su vacío. 


\section{BIBLIOGRAFÍA}

Basabe, N., Iraurgi, I., Paez, D. e Insua, P. (1996). Psicoinmunología, factores psicosociales e infección por VIH. En N. Basabe, D. Paez, R. Usieto et al. (Eds). El desafío social del SIDA, 299-350. Madrid, Fundamentos.

Basabe, N., Valdoseda, M. y Paez, D. (1993). Memoria afectiva, salud, formas de afrontamiento y afectividad. En D. Paez (Ed). Salud, expresión y represión social de las emociones, 339-377. Valencia, Promolibro.

Conde, V. y Franch, J. (1984). Escalas de evaluación conductual para la cuantificación de sintomatología psicopatológica en los trastornos ansiosos y depresivos. Valladolid, Dpto. de Psicología Médica, Universidad de Valladolid.

Echevarría, A. y Paez, D. (1989). Emociones: Perspectivas Psicosociales. Madrid, Fundamentos.

Iraurgi, I., Basabe, N., Sastre, J., Del-Romero, J., García, S., Paez, D., Usieto, R. y Rodríguez C. (1996). Factores psicosociales, intervención psicológica y respuesta inmunitaria en afectados por VIH. Presentación de un estudio. Comunicación presentada en el III Reunión Nacional sobre el SIDA, Abril, Santiago de Compostela.

Iraurgi, I. y Jiménez-Lerma, J.M. (1998). Estrategias de afrontamiento en usuarios de drogas. Psiquis, 19, 1, 20-30.
Lazarus, R.S. y Folkman, S. (1986). Estrés y procesos cognitivos. Barcelona, Martínez Roca.

Kalichman, S.C. y Sikkema, K.L. (1994). Psychological sequela of HIV infection and AIDS: Review of empirical finding. Clinical Psychology Review, 14, 7, 611-632.

Namir S, Wolcott DL, Fawzy IF, Alumbaugh J (1987). Coping with AIDS: Psychological and Health implications. Journal of Applied Social Psychology, 17, 3, 309-328.

Norusis, MJ. (1994). Statistical Package for the Social Sciences. Release 6.1. Chicago, SPSS Inc.

Paez, D. (1986). Salud mental y factores psicosociales. Madrid, Fundamentos.

Paez, D. (1993). Salud, expresión y represión social de las emociones. Valencia, Promolibro.

Spielberger, C., Gorsuch, R. y Lushene, R. (1970). Manual for the State-Trait Anxiety Inventory. Palo Alto, CA, Consulting Psychologists Press.

Taylor, S.E. (1983). Adjustment to threatening events: A theory of cognitive adaptation. Americam Psychologist, 11, 1161-1173.

Vaux, A., Phillips, J., Holly, C., Thomson, B., Williams, D. y Stewart, D. (1986). The Social Support Appraisals (SAS) Scale: Studies of reliability and validity. American Journal of Community Psychology, 14, 195-219. 